\title{
Field Spectroscopy by Optical Fibres: development of the "ALBIREO" spectrograph
}

\author{
G. Herpe ${ }^{1}$, J. Sanchez ${ }^{2}$, Ch. Vanderriest ${ }^{1}$, M. Moles ${ }^{2}$
}

\begin{abstract}
A multi-function spectrograph has been built for the new $1.52 \mathrm{~m}$ telescope in the Sierra Nevada Observatory near Granada (Spain). It uses optical fibres for both multi-object spectroscopy and integral field spectroscopy, in addition to classical imaging and long-slit modes.
\end{abstract}

A collaboration was arranged between the EROES team of the Laboratoire d'Astronomie Extragalactique et de Cosmologie (Observatoire de Meudon, France) and the Instituto de Astrofisica de Andalucia (Granada, Spain) for developping a multi-purpose spectrograph which will be permanently mounted at one of the 2 Nasmyth foci $(F / 8$, scale: 1 " $=60 \mu \mathrm{m})$ of the new $1.52 \mathrm{~m}$ telescope in the Sierra Nevada Observatory. It will be used for both stellar and extragalactic programs. Multi-object as well as integral field spectroscopy capabilities were requested. We briefly describe this instrument.

\section{Characteristics of the "ALBIREO" spectrograph}

The instrument consists in 3 main parts: the bonnette, the spatial stage and the spectrographic stage. Its detector will be a $1024 \times 1024$ CCD built by IAA.

- The "bonnette" contains a video camera movable on $\mathrm{x}, \mathrm{y}, \mathrm{z}$ axes for field acquisition and guiding. This camera can also be used to check centering in the long-slit and Argus modes. For that purpose, properly tilted reflective elements allow to see a $18 \mathrm{~mm}\left(=5^{\prime}\right)$ field around the slit or the fibre bundle entrances. The bonnette contains also a wavelenght calibration unit.

- The "spatial stage" allows to choose between 3 spectrographic modes with different spatial coverage: classical long slit of 8' length and adjustable width; multi-object spectroscopy (Medusa) using 40 optical fibres of $200 \mu \mathrm{m}$ (=3.3") diameter plugged in an aperture plate covering 20'; integral field spectroscopy (Argus) with a bundle of 243 fibres of $80 \mu \mathrm{m}(=1.3$ ") diameter covering a 12" 120 " area. A $4^{\text {th }}$ mode is direct imaging. All 4 "spatial" devices are mounted on the same sliding plate and changing the configuration is done quickly (1min) by remote control. This part contains also a filter wheel.

- The "spectrographic stage" is of classical design, with dioptric collimator $(F=336 \mathrm{~mm}, F / D=5.6)$ and camera $(F=121 \mathrm{~mm}, F / D=2)$. Reflection gratings are mounted on a wheel which holds also a mirror for the imaging mode. The pupil is $60 \mathrm{~mm}$. With the present set of gratings, the dispersions are rougly

${ }^{1}$ L.A.E.C. from the C.N.R.S. and Observatoire de Paris-Meudon, France

${ }^{2}$ I.A.A. from the C.S.I.C. Granada, Spain 
between $200 \AA / \mathrm{mm}$ and $20 \AA / \mathrm{mm}$ and the resolved element between $5 \AA$ and $0.5 \AA$ in Argus mode. The useful wavelength range is $3500-7500 \AA$.

\section{Schedule}

After completion of the mechanics and optics, Albireo is now in Granada for electronics and software integration. The first tests on the telescope are scheduled for the end of 1994 and it is expected to be commissioned in 1995. The first scientific programmes to be adressed concern the kinematics of galaxies.

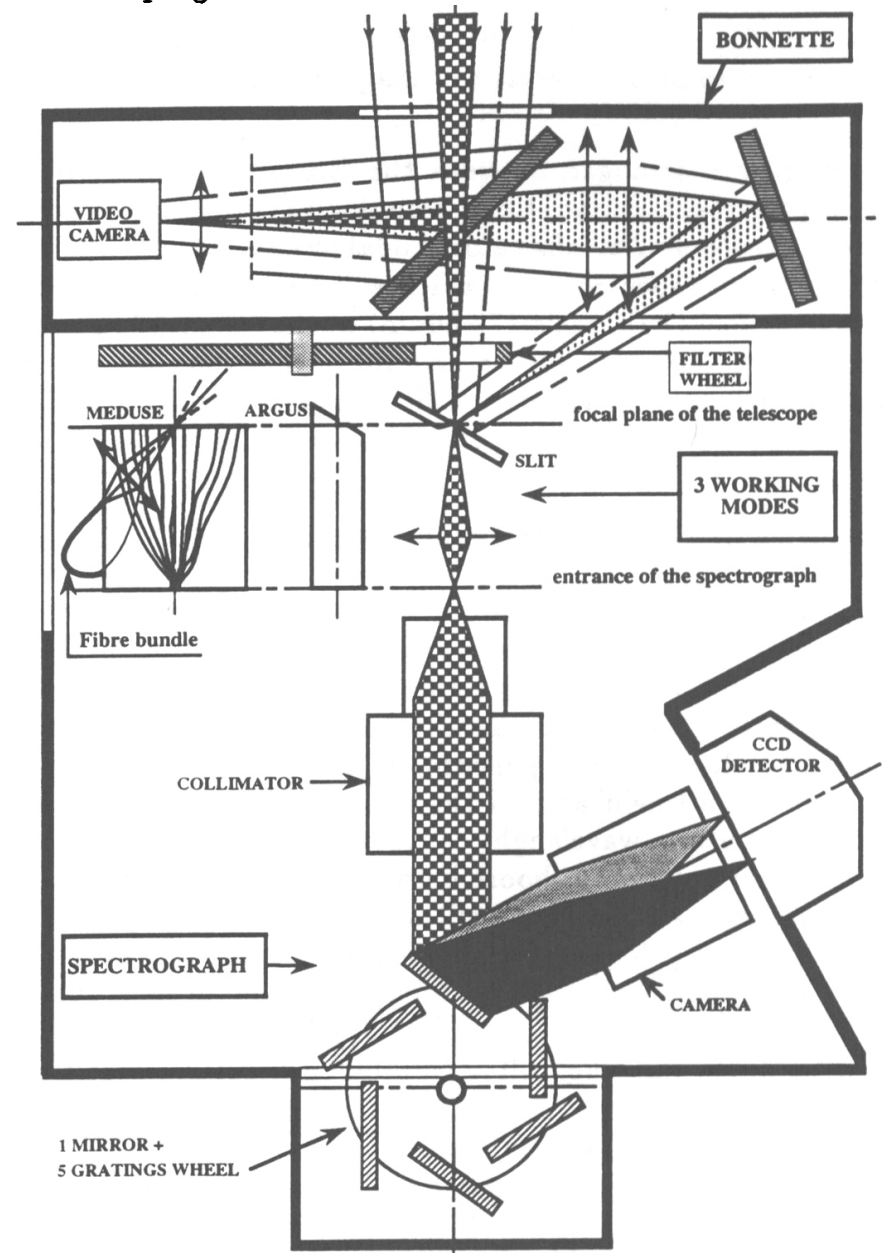

Figure 1. Principle of the "Albireo" optical fibres spectrograph

Acknowledgments. We warmly thank A. Piacentino, F. Balsamo, J.R. Fremy, the technical staff of the I.A.A., the CNRS administration for help. The development was financially supported by the I.A.A. from CSIC, Spain. 